\title{
The Totally Positive Completion Problem: The 3-by-n Case
}

https://doi.org/10.1515/spma-2020-0134

Received October 23, 2020; accepted March 24, 2021

Abstract: The 3-by- $n$ TP-completable patterns are characterized by identifying the minimal obstructions up to natural symmetries. They are finite in number.

Keywords: Completion problem, Initial minors, Totally positive matrix

MSC: 15A83, 15B48

\section{Introduction}

An $m \times n$ real matrix A is called totally positive (TP) if each of its minors (determinants of square submatrices) is positive. To verify that a matrix A is TP, only the contiguous minors of A need to be checked; this is known as Fekete's Theorem [2]. Better yet, only the initial minors, contiguous minors in which the first row or column is included, need to be checked. A useful reference about total positivity can be found in [2].

A partial matrix is a rectangular array in which some entries are specified and the remaining, unspecified entries are free to be chosen (from an agreed upon set). A completion of a partial matrix is a choice of values for the unspecified entries, resulting in a conventional matrix. The TP completion problem asks which partial matrices have a TP completion. Of course, an obvious necessary condition that a partial matrix have a TP completion is that it be partial TP: all specified minors (i.e. minors determined by specified entries) must be positive. In general, this necessary condition is not sufficient; completability depends on the pattern of the specified entries and their values. A pattern is simply an inventory of the positions of specified entries. For example, we denote the unspecified entries in a pattern with "?” and the specified entries with “*”, as in

$$
\left[\begin{array}{lll}
* & ? & * \\
? & * & * \\
* & * & ?
\end{array}\right] .
$$

It is known [4] that for each pattern, there is a finite list of polynomial conditions in the specified entries that determine whether the data permit a TP completion. However, it is generally very difficult to find an efficient list of conditions. Of course, such a list may be taken to include the requirement of partial total positivity, and for some patterns, these are the only conditions! We call these the TP-completable patterns. For example, any 2-by- $n$ pattern is TP-completable, but already in the 3-by-3 case, we can find several patterns that are not TP-completable, such as (1).

D. Carter: Department of Mathematics, Princeton University, Princeton, NJ 08544

K.E. DiMarco: Department of Mathematics, Bucknell University, Lewisburg, PA 17837

C.R. Johnson: Department of Mathematics, College of William and Mary, Williamsburg, VA 23187-8795, USA, E-mail: crjohn@wm.edu

*Corresponding Author: L. Wedemeyer: Department of Mathematics, Duke University, Durham, NC 27708,

E-mail: logan.wedemeyer@duke.edu

Z. Yu: Department of Mathematics, University of Florida, Gainesville, FL 32601, E-mail: zonshenyu@ufl.edu 
The 3-by-3 TP-completable patterns were characterized in [5]. In addition, there have been other particular results about TP-completable patterns $[1,5]$.

Our purpose here is to determine the 3-by- $n$ TP-completable patterns. We do this by describing the $o b$ structions, the non-TP-completable patterns, in particular the "minimal" ones. Fortunately, they are finite in number. For the general problem, it is an interesting open question whether the minimal obstructions are finite in number.

In the next section, we assemble several known technical facts and new observations that we will need, after establishing convenient notation. In section 3, we list the minimal obstructions and prove they are not TP-completable. We note that they are all minimal, as well. In section 4, we prove that this list of minimal obstructions is complete, up to the symmetries we also identify.

\section{Basic Results}

We begin with some basic results regarding the completability of patterns.

We refer to the following result as the ratio theorem.

Theorem 1. A 2-by-n matrix $A=a_{i j}$ with positive entries is TP if and only if $\frac{a_{1 i}}{a_{2 i}}>\frac{a_{1(i+1)}}{a_{2(i+1)}}$ for all $1 \leq i \leq n-1$

Proof. By Fekete's Theorem [1], $A$ is totally positive if and only if each contiguous minor of $A$ is positive. All 1 - by - 1 minors are positive by assumption. Each contiguous 2-by-2 minor of $A$ is positive if and only if $a_{1 i} a_{2(i+1)}>a_{2 i} a_{1(i+1)}$ for all $1 \leq i \leq n-1$. Rearranging, we find that $A$ is totally positive if and only if $\frac{a_{1 i}}{a_{2 i}}>\frac{a_{1(i+1)}}{a_{2(i+1)}}$ for all $1 \leq i \leq n-1$, as desired.

It immediately follows that each 2-by- $n$ partial TP matrix is TP-completable, and therefore all $2-$ by $-n$ patterns are TP-completable.

A 3-by- $n$ partial TP matrix with 1 unspecified entry is always TP-completable [1]. Furthermore, by Theorem 3.1 of [3], we have:

Theorem 2. Let $P$ be a 3-by- $n$ pattern with all of its unspecified entries in the same row or column. Then, $P$ is TP-completable.

The following theorem is from [2]:

Theorem 3. Let $A$ be a $m$-by-n matrix, $P_{1}$ be the $m$-by-m reverse identity matrix (i.e. matrix with 1's on the antidiagonal and $O$ 's everywhere else), and $P_{2}$ be the $n$-by-n reverse identity matrix. Then, $A$ is TP if and only if $P_{1} A P_{2}$ is TP.

We refer to $P_{1} A P_{2}$ as the forwards-backwards symmetry of $A$. The theorem implies the set of TP matrices is closed under forwards-backwards symmetry. We may also apply the forwards-backwards symmetry to patterns. A pattern is TP-completable if and only if it is TP-completable after applying forwards-backwards symmetry.

The following lemma from [1] (Lemma 2.4) allows us to border a partial TP matrix to get a larger matrix while maintaining partial TP.

Lemma 1. Let $A$ be an $m$-by-n partial TP matrix. Then there exist positive vectors $x, u, v, w$ such that $[A \mid x]$, $[u \mid A],\left[\begin{array}{c}A \\ v\end{array}\right]$, and $\left[\begin{array}{l}w \\ A\end{array}\right]$ are all partial TP. 
Of course, if we want to border with a column or row that is not fully specified, we may just apply the above lemma, and unspecify some entries.

There are infinitely many 3-by- $n$ non TP-completable patterns, but many of them are due to a smaller subpattern that is not TP-completable.

Lemma 2. Let a pattern $P$ be not TP-completable. Let $P^{\prime}$ be a pattern that contains $P$ as a contiguous subpattern. Then $P^{\prime}$ is also not TP-completable.

Proof. Let $A$ be a partial TP matrix of pattern $P$ that has no completion. By Lemma 1, we may border $A$ and remain partial TP. Do so until we get a partial TP matrix $A^{\prime}$ with the same dimensions as $P^{\prime}$. Now, unspecify any entries that are unspecified in $P^{\prime}$. The result is a partial TP matrix of pattern $P^{\prime}$. If this were TP-completable, we would have a completion for $A$, a contradiction.

Also,

Lemma 3. Let a pattern $P$ be not TP-completable. Let $P^{\prime}$ be a pattern obtained from $P$ by inserting a column with 2 or fewer specified entries. Then, $P^{\prime}$ is also not TP-completable.

Proof. We prove the case of exactly 2 specified entries; if there are less one may first insert a column with 2 specified entries and then make one of the two unspecified after the fact.

Let $A$ be a partial TP matrix of pattern $P$ that is not TP-completable. Insert a new column in $A$ to get a partial matrix $A^{\prime}$ that is of pattern $P^{\prime}$. We still need to specify the entries in the new column. Consider the 2-by- $n$ partial TP submatrix of $A^{\prime}$ corresponding to the two rows the two specified entries in the new column are in. Complete this matrix to be TP, treating the 2 specified entries as unspecified ones. Then just unspecify all the entries that are unspecified in pattern $P^{\prime}$. Because the only completed minors are in that 2-by- $n$ submatrix, and we unspecified some data in a TP matrix, $A^{\prime}$ is partial TP. Hence, we have partial TP data for $A^{\prime}$, which if TP-completable, would yield a completion for the partial TP data in $A$. But such a completion does not exist, so $P^{\prime}$ is also not TP-completable.

Hence, it makes sense to only study the obstructions that are "minimal" in light of the previous two lemmas. Establishing what exactly minimal entails is key to a full classification of the 3 -by- $n$ non-completable patterns.

Definition 1. A 3-by-n obstruction $P$ is said to be minimal if $P$ contains no contiguous proper subpattern that is an obstruction, and no subpattern obtained from $P$ by removing columns with 2 or fewer specified entries is an obstruction.

The following lemma allows for a more concise presentation of 3-by- $n$ patterns.

Lemma 4. Let $P$ be a 3-by-n pattern with column $i$ having $O$ or 1 specified entries. Set $P^{\prime}$ to be the pattern obtained from $P$ by removing column $i$. Then, $P$ is TP-completable if and only if $P^{\prime}$ is.

Proof. We consider the case in which column $i$ has 1 specified entry, the case of 0 specified entries follows similarly. First, suppose pattern $P$ is TP-completable. Let $A^{\prime}$ be a partial TP matrix of pattern $P^{\prime}$, and insert a column with 1 specified entry after column $i-1$ to get a partial matrix $A$ of pattern $P$. Choose any positive value for the specified entry in column $i$ of $A$. $A$ is partial TP because no minors except the 1-by-1 minor corresponding to said entry were completed. Complete $A$ using pattern $P$ 's completability. Removing column $i$ provides a completion of $A^{\prime}$.

Now, suppose pattern $P^{\prime}$ is TP-completable. Let $A$ be a partial TP matrix of pattern $P$. Complete everything but column $i$ by using the completion for that data in $P^{\prime}$. Now, we have a partial TP matrix with 2 unspecified entries in the same column, which is TP-completable by Theorem 2. 
This lemma shows that columns with 1 or fewer specified entries are essentially irrelevant to completability for 3-by- $n$ patterns. Hence, from now on, unless otherwise specified, we assume all patterns have no columns with 1 or fewer specified entries, since these cannot be minimal obstructions.

Moreover, this means there are only 4 possibilities for the columns of any 3-by- $n$ pattern $P$, with three possibilities if there are 2 specified entries, and one possibility if the column is fully specified. Hence, for convenience sake, we write 0 in place of a fully specified column, 1 in place of a column in which the unspecified entry is in row 1,2 if it is in row 2 , and 3 if it is in row 3 . In this way, we identify 3 -by- $n$ patterns to words over the alphabet $\{0,1,2,3\}$. As an example, the pattern

$$
\left[\begin{array}{lllll}
* & * & * & * & ? \\
* & ? & * & * & * \\
* & * & * & ? & *
\end{array}\right]
$$

can be concisely expressed as 02031 . We may also write that a column is type $0,1,2$, or 3 , with the same meaning as above. We will freely use this notation from now on.

The idea of forwards-backwards symmetry also translates nicely into this new language. Applying the forwards-backwards symmetry to a pattern is equivalent to reversing the word and replacing each letter $n$ by $-n$ mod 4. For instance, 02031 becomes 31020 after application of the forwards-backwards symmetry. We will to this process as reverse complementing a word. A pattern is TP-completable if and only if its reverse complement is.

\section{The list of minimal obstructions}

In Table 1 is the list of all 3-by- $n$ minimal obstructions up to symmetry, shown with partial TP data that does not admit a TP completion. The left table has the distinct reverse complement pairs of minimal obstructions; data is presented for the first pattern listed. On the right, the four patterns are their own reverse complement.

Verifying that the patterns with the given data are not TP-completable is straightforward. The patterns $210,213,230,231,3100$, and 3200 can be verified to be non-completable by considering only a single unspecified entry and the minors it completes. For 210, 213, 230, and 231, for the two 2-by-2 minors that $a_{21}$ completes to be positive, $a_{21}$ must both be greater than and less than one, which is impossible. For 3100 or 3200, the topleft 2-by-2 minor forces $a_{12}<1$ or $a_{22}>1$, respectively, and the rightmost 3-by-3 minor forces the opposite inequality, so they are impossible to complete.

The patterns 2112, 31021, 31031, 32021, and 31130 can be verified to be non-completable by considering two unspecified entries. For 2112, 2-by-2 minors force both $a_{21}>1$ and $a_{24}<1$, but this makes the minor consisting of rows 1 and 2 and columns 1 and 4 negative. For 31021, 31031, and 32021, 2-by- 2 minors force respectively $a_{12}<1$ and $\frac{16}{3}>a_{24}>5, a_{12}<1$ and $\frac{15}{2}<a_{34}<8$, and $a_{22}>1$ and $a_{24}>5$. In all cases, this makes the 3-by-3 minor consisting of columns 2, 3, and 4 to be negative. Finally, for 31130, 2-by-2 minors force $a_{12}<1$ and $\frac{14}{3}>a_{34}>4$, which makes the 3-by-3 minor consisting of columns 2, 4, and 5 negative. Similarly, the pattern 311331 can be verified to be non-completable by noting that 2-by-2 minors force $a_{12}<1$, $\frac{14}{3}>a_{34}>4$, and $6<a_{35}<7$, and $12<3 a_{34}<2 a_{35}<14$. This makes the 3 -by-3 minor consisting of columns 2 , 4, and 5 negative. Finally, for 0130, a 2-by- 2 minor forces $a_{12}>1 / 2$. Then, the rightmost 3-by-3 minor forces $-a_{33}\left(3 a_{12}-1\right)+14 a_{12}-5>0$, which given the restriction on $a_{12}$ is equivalent to $a_{33}<\left(14 a_{12}-5\right) /\left(3 a_{12}-1\right)$. This makes the leftmost 3-by-3 minor less that $-2\left(1-2 a_{12}\right)^{2} /\left(3 a_{12}-1\right)$, which is negative since $a_{12}>1 / 2$.

We note that deleting a column from any of the patterns on the above list results in a TP-completable pattern. This clearly holds for the 3-by-3 patterns, as all 2-by-3 and 1-by-3 patterns are TP-completable and hence no proper subpattern could be an obstruction. None of the remaining patterns contain any contiguous subpatterns that are obstructions, and the removal of any type 1, 2, or 3 column results in a TP-completable pattern. 
Table 1: List of 3-by- $n$ minimal obstructions up to symmetry

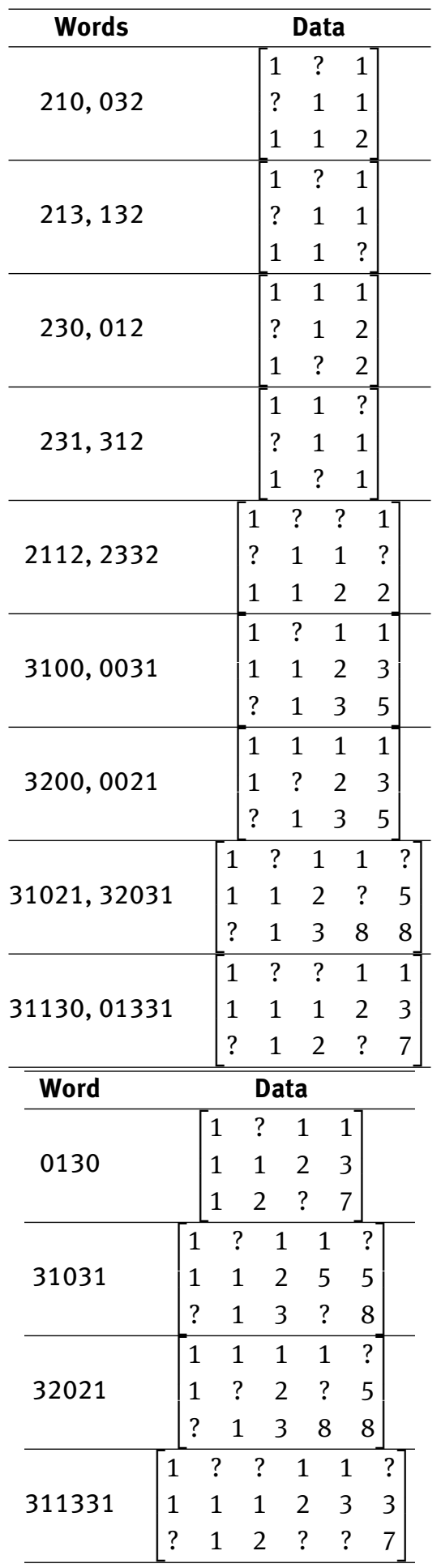

\section{Proof the list is complete}

It remains to show that there are no other minimal obstructions. We proceed by restricting the search space for potential minimal obstructions until the resulting list is manually completable. First, we establish restrictions 
on the patterns that could be unlisted minimal obstructions. Then, we identify a subset of the remaining possibilities as 'maximal', and complete those patterns. Finally, we conclude that all remaining potential minimal obstructions are completable.

\subsection{Reductions}

A pattern $P$ that we need to check to see if it is a minimal obstruction or not is subject to the following constraints, proven later:

1. No two consecutive columns of $P$ are both fully specified, except possibly the first two or last two columns.

2. No three consecutive columns of $P$ are identical.

3. The two columns before any fully specified column cannot be identical. The same holds for the two columns after any fully specified column.

4. Between any two fully specified columns, there are at least two distinct types of columns.

5. As a word, $P$ does not begin with $x x$ or $x 0$, and $P$ does not end with $x x$ or $0 x$, where $x \in\{1,2,3\}$.

Moreover, all patterns that do not satisfy the above constraints can be reduced to a smaller pattern which does satisfy the constraints. The exact way the reductions are made is given below, with the justifications.

Lemma 5 (Constraint 1). Let $P$ be a 3-by-n pattern, such that columns $i, i+1$ are fully specified. Let $P_{1}$ be the subpattern consisting of columns 1 through $i+1$ of $P$, and $P_{2}$ be the subpattern consisting of columns $i$ to $n$ of $P$. If $P_{1}$ and $P_{2}$ are TP-completable, so is $P$. Conversely, if one of $P_{1}$ or $P_{2}$ is an obstruction, so is $P$.

Proof. Let $A$ be a partial TP matrix of pattern $P$, and complete the entries in columns 1 through $i+1$ and in columns $i$ through $n$ using the completability of $P_{1}$ and $P_{2}$ respectively. There is no problem because the overlap between those sets of columns occurs only in the two fully specified columns. Then, to show $A$ is TP, we only need to check its initial minors. But the initial minors all occur entirely in either columns 1 through $i+1$ or in $i$ through $n$ (because we only need to check up to 3 contiguous columns), and hence are positive. So, $A$ is TP. The converse holds immediately, as $P$ contains a obstruction contiguously, and hence is itself an obstruction.

Hence, if $P$ is not TP-completable, then one of $P_{1}$ and $P_{2}$ is also an obstruction, so $P$ is not minimal. The only exception is if columns $i$ and $i+1$ are the first or last two columns, as then one of $P_{1}$ or $P_{2}$ is all of $P$, and the other is just 2 fully specified columns. This lemma doesn't allow for any reductions in this case.

Lemma 6 (Constraint 2). Let $P$ be a 3-by-n pattern, and let columns $i, i+1, i+2$ all be identical. Set $P^{\prime}$ to be the subpattern of $P$ obtained by removing column $i+1$. Then, $P$ is TP-completable if and only if $P^{\prime}$ is.

Proof. The cases where columns $i, i+1, i+2$ have 0 unspecified entries is essentially covered by the previous lemma. Hence, we only need to prove this lemma for the cases where columns $i, i+1, i+2$ are type 1,2 , or 3 . We will only do the case where they are type 3 , the other cases follow in the same way.

Suppose pattern $P$ is TP-completable. Let $A^{\prime}$ be a partial TP matrix of pattern $P^{\prime}$, and $A$ be a partial matrix of pattern $P$ obtained by inserting a column between columns $i, i+1$ in $A^{\prime}$. We still need to specify the entries in column $i+1$ of $A$ to match pattern $P$. Take any rational in the interval $\left(\frac{a_{1, i+2}}{a_{2, i+2}}, \frac{a_{1, i}}{a_{2, i}}\right)$, and set its numerator to be $a_{1, i+1}$ and denominator to be $a_{2, i+1}$. By the ratio theorem, we are guaranteed that the submatrix of $A$ corresponding to rows 1 and 2 is TP, and because those were the only new minors completed after the entries in column $i+1, A$ is partial TP. Then, just complete $A$ and we get a completion of $A^{\prime}$.

Suppose pattern $P^{\prime}$ is TP-completable. Let $A$ be a partial TP matrix of pattern $P$, and complete everything but column $i+1$ using the completability of $P^{\prime}$. $A$ is still partial TP at this point. To see this, we only need to verify the 2-by- $n$ submatrix corresponding to rows 1 and 2 is TP. But for any $j<i$, because all but column $i+1$ were completed to be TP, we have $\frac{a_{1, j}}{a_{2, j}}>\frac{a_{1, i}}{a_{2, i}}$, and for any $k>i+2$, we have $\frac{a_{1, i+2}}{a_{2, i+2}}>\frac{a_{1, k}}{a_{2, k}}$. Moreover, because the 
data was partial TP to begin with, we have $\frac{a_{1, i}}{a_{2, i}}>\frac{a_{1, i+1}}{a_{2, i+1}}>\frac{a_{1, i+2}}{a_{2, i+2}}$. Transitivity and the ratio theorem shows that the 2-by- $n$ submatrix corresponding to rows 1 and 2 is indeed TP. Hence, $A$ is now a partial TP 3-by- $n$ matrix with 1 unspecified entry. These are all TP-completable, so $A$ is TP-completable.

Hence, when we see three or more identical columns in a row, it can be replaced by just two copies of that column, which is the second constraint. The following lemmas are simple variants on the one above.

Lemma 7 (Constraints 3 and 4). Let $P$ be a 3-by-n pattern, such that there is a contiguous subpattern of the form $0 x x, x x 0$, or $0 x 0$ for $x=1,2$, or 3 in columns $i, i+1, i+2$. Set $P^{\prime}$ to be the subpattern of $P$ obtained by removing column $i+1$. Then, $P$ is TP-completable if and only if $P^{\prime}$ is.

Proof. We consider the case $x=3$, the other cases follow identically. First, suppose $P$ is TP-completable. Take any partial TP data for $P^{\prime}$, and insert into the corresponding location in $P$ to get a partial matrix $A$, except that we need to choose the values in $a_{1, i+1}$ and $a_{2, i+1}$. We can choose these values so that $A$ is partial TP, as in Lemma 6. Then, we just complete $A$ (as it is of pattern $P$ ) to obtain a completion for that data of pattern $P^{\prime}$.

Now, suppose $P^{\prime}$ is TP-completable. Let $A$ be a partial TP matrix of pattern $P$. Complete all columns of $A$ except $i+1$ by the completability of $P^{\prime}$. We claim $A$ is still partial TP. If so, we are done, as all 3-by- $n$ partial TP matrices with 1 unspecified entry is TP-completable. We only need to verify the 2-by- $n$ submatrix corresponding to rows 1 and 2 is TP. But for any $j<i$, because $A$ without column $i+1$ was completed to be TP, we have $\frac{a_{1, j}}{a_{2, j}}>\frac{a_{1, i}}{a_{2, i}}$, and for any $k>i+2$, we have $\frac{a_{1, i+2}}{a_{2, i+2}}>\frac{a_{1, k}}{a_{2, k}}$. Moreover, because the data was partial TP to begin with, we have $\frac{a_{1, i}}{a_{2, i}}>\frac{a_{1, i+1}}{a_{2, i+1}}>\frac{a_{1, i+2}}{a_{2, i+2}}$. Transitivity and the ratio theorem shows that the 2-by- $n$ submatrix corresponding to rows 1 and 2 is indeed TP.

This justifies constraints 3 and 4 . For constraint 4, note that patterns of the form ...0x...x0... (where between the 0 's we have all $x$ 's) may first be simplified to ..0x0..., then to just ...00... using this lemma, where $x \in$ $\{1,2,3\}$.

Lemma 8 (Constraint 5, Part 1). Let $P$ be a 3-by-n pattern of the form $x 0 \ldots$ (or ...0x), and let $P^{\prime}$ be the subpattern of $P$ obtained by removing the first (last) column. Then, $P$ is TP-completable if and only if $P^{\prime}$ is.

Proof. Let $P$ be of the form $x 0$.... The reverse case follows similarly. First, suppose $P$ is TP-completable. Let $A$ be a partial TP matrix of pattern $P^{\prime}$. By Lemma 1, we can extend $A$ by a fully specified column on the left and remain partial TP. Then, just unspecify the entry that is supposed to be unspecified. This is still partial TP. Now $A$ is a partial TP matrix of pattern $P$, which is TP-completable. So complete it to obtain a completion for the original matrix $A$. Hence $P^{\prime}$ is TP-completable.

Now, suppose $P^{\prime}$ is TP-completable. We will take $x=3$, but the other cases follow similarly. Let $A$ be a partial TP matrix of pattern $P$. Complete columns 2 through $n$ to be TP as $P^{\prime}$ is TP-completable. As before, it suffices to show $A$ is still partial TP, as we can complete 3-by- $n$ partial TP matrices with 1 unspecified entry. We know that for $k>2, \frac{a_{1,2}}{a_{2,2}}>\frac{a_{1, k}}{a_{2, k}}$, as columns 2 through $n$ are TP. Also, $\frac{a_{1,1}}{a_{1,2}}>\frac{a_{1,2}}{a_{2,2}}$ as the data was originally partial TP. Hence, by the ratio theorem, the 2-by- $n$ submatrix of $A$ in rows 1 and 2 is TP. Because no fully specified 3 by 3 minors involve column 1, $A$ is partial TP, as needed.

Lemma 9 (Constraint 5, Part 2). Let $P$ be a 3-by-n pattern of the form $x x \ldots$ (...xx) for $x=1,2$ or 3. Let $P^{\prime}$ be the subpattern of $P$ obtained by removing the first (last) column. Then, $P$ is TP-completable if and only if $P^{\prime}$ is.

Proof. Essentially identical to the proof for the previous lemma.

These lemmas can be thought of as "reductions," where a pattern $P$ can potentially be made smaller ("reduced") without impacting whether or not it is TP-completable.

Definition 2. A pattern $P$ is said to be fully reduced if all five constraints are satisfied. That is, none of the above reductions can be made. 
Note that a minimal obstruction must be fully reduced. Suppose $P$ is an obstruction that is not fully reduced. If constraint 1 is not satisfied, $P$ contains another obstruction contiguously. If any of the other four constraints are not satisfied, $P$ contains an obstruction after removal of a column with 2 specified entries. Either way, $P$ is not minimal.

Hence, any minimal obstructions that may have been missed will occur among the fully reduced patterns which avoid containing any of the known minimal obstructions contiguously, after the removal of (possibly zero) columns with 2 specified entries. Call this set of patterns $S$. We will have proved that our list of 3-by- $n$ minimal obstructions is complete if we show all patterns in $S$ are TP-completable.

We say that a fully reduced pattern $P$ is maximal if the insertion of a type 1,2 , or 3 column anywhere into $P$ results in a pattern which may be reduced.

Now, we find all maximal patterns in $S$, broken down by how many fully specified columns they have.

\subsection{No fully specified columns}

For a pattern $P$ with no fully specified columns to be in $S$, it must not contain the known obstructions 213 , $132,231,312,2112,2332$, or 311331 as a subpattern.

If $P$ begins with a 2, then because it needs to avoid 213 and 231, $P$ must be a word over the alphabet $\{1,2\}$ or $\{2,3\}$. The only constraints left on $P$ are the reductions, and avoiding 2112 or 2332 . So, if we start with a 21, we are free to append a 22, because it does not contribute to the creation of any 2112 patterns. From here, we are forced to append a 1 because we can't have 3 identical consecutive columns. Thus far, we have 21221. We can't append a 2 anymore, as we would end up with a 2112, so we must append another 1, for 212211. However, after appending the 1 , there is nothing else we can append, so that we would be ending with a 11, which does not satisfy constraint 5. Hence, we end at 21221. The case we start with a 23 is identical, yielding 23223.

If $P$ begins with a 1, then we know that it cannot be followed by another 1 . If the pattern begins with a 12 , we know that by the previous analysis that the remaining word can only contain $1 \mathrm{~s}$ and $2 \mathrm{~s}$ or $2 \mathrm{~s}$ and $3 \mathrm{~s}$. If we only have $1 \mathrm{~s}$ and $2 \mathrm{~s}$, this essentially reduces to the case where $P$ began with a 21 , except we are allowed to turn the first 2 into a 22 because the 22 is no longer at the start of the word. Hence, the longest pattern here is 1221221. On the other hand, if we have 12 followed by a word over $\{2,3\}$, we must take care to avoid 132 . We can append another 2, as it makes no new contribution to any 132 patterns. Afterwards, we must append a 3 , to get 1223. This is as long as we can go, for if we append another 2, we get a 132, and if we append another 3 , it cannot be followed by anything, which would result in a possible reduction due to the 33 at the end.

If the pattern begins with a 13 , then $P$ contains no type 2 columns, because it needs to avoid 132 . So, we have a word over the alphabet $\{1,3\}$, beginning with 13 , and we need to avoid 311331 . Because we have a 3 already, we need to avoid 11331 in the remaining word, after 13. So, we should append a 3. Then, we have two 3s in a row already, so we must include a 1 . The word is now 1331, and we must avoid 1331 in any future appending. Continuing as before, we eventually get to 13313311311, and we must avoid 31 in any further appending. With two 1s in a row, we have to add a 3 to get 133133113113. We can't append a 1, else we contain 311331. But if we append a 3, then we wouldn't be able to append anything after the 3, and hence the pattern would end in a 33, which is not allowed either. So, 133133113113 is the longest possible.

If $P$ begins with a 3, the analysis proceeds in a similar fashion. If it is followed by a 2, we get 3223223 or 3221. If it is followed by a 1, we have a 31 and anything we append must avoid 1331. So, at best we can do 3133113113.

So, in total, the maximal patterns obtained from each case are 212211, 23223, 1221221, 1223, 133133113113, 3223223,3221 , and 3133113113. But some of these are contained in each other, and hence aren't actually maximal. So the list reduces to 1223, 3221, 1221221, 3223223, and 133133113113. We are guaranteed we have all maximal patterns with no fully specified columns because in each case of the first two columns, there ended up only being one or two choices for each following column, and whenever there were two choices, one of the choices ends up bringing the restrictions to the same spot as in the other choice, just after adding extra columns. 


\subsection{One fully specified column}

We consider 3-by- $n$ patterns of the form

$$
\left[\begin{array}{lll} 
& * & \\
A & * & B \\
& * &
\end{array}\right]
$$

where $A, B$ are patterns with no fully specified columns. The known minimal obstructions 31021, 31031, 32021 and 32031 are the only restrictions that involve both $A$ and $B$. Hence, we consider the following cases: $A$ contains 31 or 32 (hence $B$ does not contain 31 or 21), $B$ contains 31 or 21 (hence $A$ does not contain 31 or 32 ), or $A$ does not contain 31 or 32 and $B$ does not contain 31 or 21. Within each case, $A$ and $B$ may be constructed to be as long as possible independently of each other, and hence we can apply the same process as in the 0 fully specified columns case to find all maximal patterns.

\subsubsection{A contains 31 or 32}

Beginning with the possibilities for $A$, we need to avoid 21, 23 and 3113 as subpatterns due to the obstructions 210, 230, 31130 (these patterns would be obtained after removal of columns with 2 specified entries). We also need to avoid 213, 132, 231, 312, 2112, 2332, or 311331 in subpatterns of $A$ as before, because if they existed, these patterns would appear contiguously after removal of columns with 2 specified entries.

$A$ cannot begin with a 2, because after the 2 cannot be a 1 or a $3(210,230)$, and hence no 31 or 32 patterns will appear.

If $A$ begins with a $1, A$ cannot start with 12, because of the same reason as before. So, $A$ must begin with a 13 , and because of 132, the rest of $A$ is a word over $\{1,3\}$. $A$ must avoid 3113 and 311331 , but because 3113 is a subpattern of 311331, we only need to avoid 3113. Then, we just proceed as in the case with 0 specified columns, choosing the next letter as we go. 13 needs to avoid 113, so we put another 3, then we have to put a 1. Now we have 1331, and need to avoid 13 in the rest of the word. Then we get 1331331, and we need to avoid 3. We can't have two identical columns followed by a fully specified column, so that is the best we can do. 1331331 indeed has a 31, so this is valid.

If $A$ begins with a 3, it can begin with either a 31 or a 32. If it begins with 32, the 2 cannot be followed by anything else, so that is maximal. Else, 3 is followed by a 1, and we still need to avoid 3113. This means the rest of the word needs to avoid 13, so as in the analysis above, the longest we can go is 31331 . The maximal patterns here are 32 and 31331. But 1331331 contains 31331, so the maximal possibilities for $A$ are 32 and 1331331.

Now for $B$, it must avoid 31 and 21 because $A$ contains 31 or 32. Other than that, we need to avoid 213, 132, 231, 312, 2112, 2332, or 311331 in subpatterns of $B$. We also need to avoid 32, 12, and 1331 from 032, 012, 01331, the reverse complements of the patterns in $A$. Because $B$ needs to avoid 32 and 31, it cannot begin with a 3 .

If $B$ begins with a 2, it must go 23. Then, the need to avoid 31 and 32, as well as the fact that a pattern cannot end on two copies of the same column means that this is maximal.

If $B$ begins with a 1, it cannot be 12 , so it must be 13 . Like in the 23 case, it is maximal here. So, the maximal possibilities of $B$ are 23 or 13 .

Now, we just pair all possibilities for $A$ with all possibilities for $B$. This gives the patterns 32023,32013 , $1331331023,1331331013$.

\subsubsection{B contains 31 or 21}

If $B$ contains a 31 or $21, A$ must avoid 31 or 32 . Hence, $A$ cannot begin with a 3 . If $A$ begins with a 2, the obstructions 210 and 230 ensure that this is the longest possible pattern in this case. Finally, if $A$ begins with a 1, going 12 is as long as possible (because the two columns before a 0 can't be identical). Else, $A$ begins with a 13. The 3 cannot be followed by a 2, as 132 is a known obstruction, and it can't be followed by a 1, because 
$A$ avoids 31 . So, 13 is also as long as possible for $A$ here. The possibilities are therefore 2, 12, or 13 , and getting rid of patterns that aren't actually maximal leaves just 12 and 13 for $A$.

As for $B$, if it begins with a 2, it cannot go 23, as avoiding 231 means there would be no 31 or $21 \mathrm{~s}$ in $B$. So, $B$ must begin with 21. We cannot append a 2 or a 3 because of 12 and 213. We can't have 2 copies of a column at the end of a pattern, which means 21 is maximal.

If $B$ begins with a 3, it must be followed by a 1 because of 32. The pattern 312 that we must have a word over $\{1,3\}$ after the 31 . We need to avoid 1331 and 311331, but 1331 is a subpattern of 311331, so it suffices to avoid 1331. The process to obtain the longest possible word follows the previous reasoning, giving the final maximal pattern 3113113.

If $B$ begins with a 1, it must be followed by a 3 because of 12. The obstruction 132 means we can only follow the 13 by more 1s and 3s. We still need to avoid 1331. As always, the process is the same, giving 13113.

The possible maximal patterns for $B$ are 21, 3113113, 13113. Hence, the actual list is just 21 and 3113113. Therefore, the list of maximal patterns in this case is 12021, 1203113113, 13021, 1303113113.

\subsection{3 $A$ avoids 31, 32, $B$ avoids 31,21}

We already know the maximal patterns for $A$ and $B$ in this case. $A$ can be 12 or 13 , and $B$ can be 23 or 13 . So, the possibly maximal patterns in this case are 12023, 12013, 13023, 13013.

So, the maximal patterns from each case are 32023, 32013, 1331331023, 1331331013, 12021, 1203113113, 13021, 1303113113, 12023, 12013, 13023, and 13013. Getting rid of ones that are contained in others, we get a final list of 32023, 32013, 1331331023, 1331331013, 12021, 1203113113, 13021, 1303113113, and 12023.

\subsection{Two fully specified columns}

Here, we consider patterns of the form

$$
\left[\begin{array}{rrrrr} 
& * & & * & \\
A & * & B & * & C \\
& * & & * &
\end{array}\right]
$$

where $A, B, C$ are patterns with 0 fully specified columns.

First, we consider what $B$ can be. It cannot be one column long, as then the pattern would be reducible. It must avoid 21, 23, and 13 due to the obstructions 210, 230, and 0130. It must also avoid 12 and 32 from the reverse complements of 210 and 230. Hence, $B$ must start with a 31, if it is not empty. But after the 1 cannot be a 3 or a 2 because of 12 and 32. There can't be two of the same column before a fully specified column, so if $B$ is nonempty, it can only be 31 . However, it is possible for a maximal pattern to exist with $B$ empty, so long as either $A$ or $C$ is also empty due to constraint 1. We are left with the following possible patterns:

$$
\begin{aligned}
& P_{1}=\left[\begin{array}{lll} 
& * & * \\
A & * & * \\
& * & *
\end{array}\right], \\
& P_{2}=\left[\begin{array}{lll}
* & * & \\
* & * & C \\
* & * &
\end{array}\right], \\
& P_{3}=\left[\begin{array}{llllll} 
& * & * & ? & * & \\
A & * & * & * & * & C \\
& * & ? & * & * &
\end{array}\right]
\end{aligned}
$$

For $P_{1}, A$ needs to avoid 31, 32, 21, and 23 from the obstructions 3100, 3200, 210, 230. So, $A$ either begins with a 12 or a 13. But both of these are maximal, as neither 2 or 3 can be followed by anything but another copy of 
themselves, and we know two identical columns before a fully specified column can be reduced to just 1 . So, $A$ is 12 or 13 , and the maximal patterns are 1200,1300 .

For $P_{2}, C$ needs to avoid 31, 21, 32, and 12 from the obstructions 0031, 0021, 032, and 012. Hence, $C$ can begin with either 13 or 23. Like with the previous case, these are maximal. The maximal patterns here are 0013 and 0023.

Finally, for $P_{3}$, because of the 31 in the middle, we need to account for the obstructions 31031, 32031, and 31021. For $A$, this doesn't change anything. It still needs to avoid 31, 32, 21, 23, and hence 12 and 13 are maximal. For $C$, nothing changes either. So, the maximal patterns are 12031013, 12031023, 13031013, and 13031023. Note that these contain all of the maximal patterns in the $P_{1}$ and $P_{2}$ cases, so these are the only four that are actually maximal.

\subsection{3 or more fully specified columns}

We claim there are no new minimal obstructions in this case. Suppose we had a new minimal obstruction of the form

$$
P=\left[\begin{array}{rrrrrrrrrrrrrr} 
& * & & * & & * & & * & & * & & * & \\
A & * & B_{1} & * & B_{2} & * & \cdots & * & B_{k-1} & * & B_{k} & * & C \\
& * & & * & & * & & * & & * & & * &
\end{array}\right]
$$

where $k \geq 2$ and $A, B_{1}, \ldots, B_{k}, C$ all have no fully specified columns. Each $B_{i}$ has at least two fully specified columns on one side of it, and at least one on the other side.

Suppose $B_{i}$ has two fully specified columns on the right, and one on the left. Then, it must avoid 31, 32, $13,21,23$, and 12 from the obstructions 3100, 3200, 0130, 210, 230, and 012. Hence, it can only be at most one column long. Now, suppose $B_{i}$ has two fully specified columns on the left, and one on the right. It must avoid $31,21,13,23,12$, and 32 from the obstructions $0031,210,0130,230,012$, and 032 . So it too can be only at most one column long.

Hence, all $B_{i}$ are either 0 or 1 columns long. But if any $B_{i}$ is 1 column long, $P$ would not be fully reduced by constraint 4, and if any $B_{i}$ is empty, $P$ would not be fully reduced by constraint 1 , so $P$ cannot be fully reduced and thus is not in $S$.

\subsection{The maximal patterns}

The final list of maximal patterns is: $1223,3221,1221221,3223223,133133113113,32023,32013,1331331023$, $1331331013,12021,1203113113,13021,1303113113,12023,12031013,12031023,13031013$, and 13031023. Note that all patterns in $S$ are contained in some maximal pattern here. Either a pattern is maximal, in which case it is in the list, or it is not maximal, and hence can have columns inserted with two fully-specified entries to become a maximal pattern in the list. Finally, by the contrapositive to Lemma 3, this means that if we show all of the maximal patterns here are TP-completable, all patterns in $S$ are TP-completable, and hence the list of minimal obstructions is complete.

\subsection{The completions}

Thanks to the forwards-backwards symmetry, we can reduce the list of patterns we need to complete down to the following twelve patterns: 1223, 3221, 1221221, 133133113113, 12021, 13021, 1331331023, 1331331013, 12023, 12031013, 12031023, and 13031013.

Before discussing the completability of these patterns, we mention the following important lemma: 
Lemma 10. Let $P$ be a 3-by- $n$ pattern of the form $\left[\begin{array}{ccc} & * & \\ A & * & B \\ & *\end{array}\right]$, where A has no fully specified columns (but

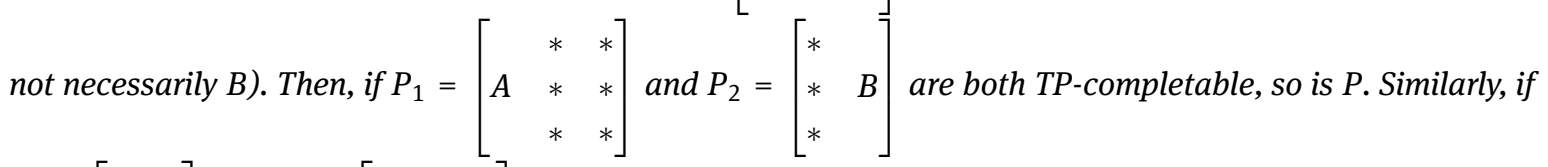

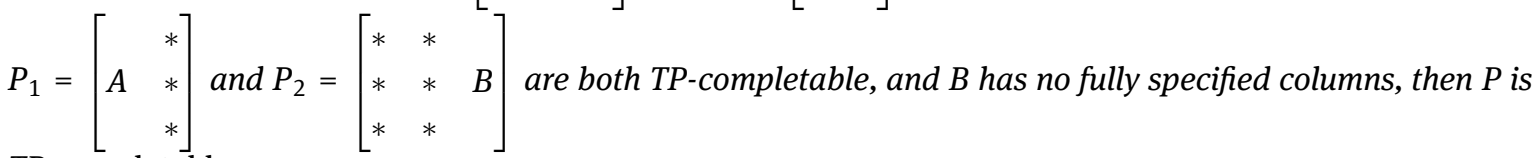
TP-completable.

Proof. We prove the first case; the other one is symmetrical. In $P$, complete $B$ according to the completion of $P_{2}$. Then, taking the first column of $B$ as specified, along with $A$ and the fully specified column, we have a pattern of the form $P_{1}$. This is partial TP because no 3 by 3 minors are fully specified (as $A$ has no fully specified columns). Now we can complete $A$, which completes $P$.

Finally, the completability of all 3-by-3 and 3-by-4 patterns with one and two unspecified entries was characterized in [1].

Theorem 4. All patterns in S are TP-completable.

Proof. By previous discussion, we need only to complete the maximal patterns of $S$ up to symmetry, which are 1223, 3221, 1221221, 133133113113, 12021, 13021, 1331331023, 1331331013, 12023, 12031013, 12031023, and 13031013. We use Lemma 10 and 3-by-3 and 3-by-4 results from [1] extensively.

- 1223: The $(1,1)$ and $(3,4)$ entries enter positively into all 2-by-2 minors, and hence can be chosen to be large and maintain partial TP. Then, we need to complete a partial TP matrix of pattern 0220, which is possible by Theorem 2 .

- 3221 Note that the $(1,4)$ and $(3,1)$ entries enter negatively into all 2-by-2 minors that they are in. As all of the specified entries are positive, we may choose these two entries to be arbitrarily close to zero, so that all 2-by-2 minors they complete are still positive. So, we complete the $(1,4)$ and $(3,1)$ entries in that fashion. Because we only completed those two entries, no 3-by-3 minor was completed, and the matrix is still partial TP. Now, we need to complete a partial TP matrix of pattern 0220 like above, which we can do.

- 1221221: Complete the 1 in the middle to get 1220221 . We are done if 1220221 is TP-completable. But this is TP-completable if and only if 12021 is. 1200 and 021 are TP-completable, and therefore 12021 is TPcompletable.

- 133133113113: First, complete a column in the middle (using 2-by- $n$ completability in rows 1 and 2) to get 133033113113. Now, reducing this pattern, we find that 133033113113 is TP-completable if and only if 1303113113 is. This is TP-completable if 1300 and 03113113 both are. 1300 is known to be TP-completable. We will prove 03113113 is TP-completable by proving its reverse complement, 13313310, is TP-completable. Note that if we can complete the fourth column while remaining partial TP, we will be done if 13303310 is TP-completable. Reducing shows that 13303310 is TP-completable if and only if 130310 is TP-completable, and this last pattern is TP-completable if 1300 and 0310 are TP-completable, both of which are known to be TP-completable. Hence, we will be done if we can complete the fourth column of 13313310 and remain partial TP. By scaling of rows and columns, without loss of generality we are trying to complete the fourth column in the following partial matrix:

$$
\left[\begin{array}{llllllll}
? & * & a & ? & b & * & ? & 1 \\
1 & 1 & 1 & 1 & 1 & 1 & 1 & 1 \\
* & ? & ? & c & ? & ? & * & 1
\end{array}\right]
$$


Here, ? denotes unspecified entries, while $a, b, c$ and all the $*$ entries are specified (as before, the $*$ is a placeholder for a specified entry). Because our data is partial TP, note that $a>b>1$, while $c<1$. Now, set the $(1,4)$ entry to be any value in the range $(b, a)$. This ensures, by the ratio theorem, that all completed minors in the first two rows are positive. The only other completed minor is in rows 1 and 3, columns 4 and 8. But this is positive if the $(1,4)$ entry is larger than $c$. Because $b>1$ and $c<1$, it is indeed positive. Hence, we may complete the $(1,4)$ entry and remain partial TP, and we are done.

- 12021: This was done when we completed 1221221.

- 13021: If 1300 and 021 are TP-completable, 13021 is TP-completable, and both are TP-completable.

- 1331331023: If 13313310 and 0023 are TP-completable, 1331331023 is TP-completable. It is known 0023 is TP-completable. For 13313310, we showed above it is TP-completable.

- 1331331013: This is TP-completable if 13313310 and 0013 are both TP-completable, and we showed 13313310 is TP-completable in the previously, and 0013 is known to be TP-completable.

- 12023: This is TP-completable if 1200 and 023 are both TP-completable, and both are.

- 12031013: This is TP-completable if 1200 and 031013 are both TP-completable. We know 1200 is TPcompletable, and showed 031013 is TP-completable above by showing its reverse complement 130310 is completable.

- 12031023: This is TP-completable if 1200 and 031023 are both TP-completable. 1200 is known to be TPcompletable. Once again, 031023 is TP-completable if 0310 and 0023 are both TP-completable, and both are.

- 13031013: This is TP-completable if 1300 and 031013 are both TP-completable. We know 1300 is TPcompletable, and showed 031013 is TP-completable above by showing its reverse complement 130310 is completable.

Hence, all maximal patterns of $S$ are TP-completable, and the proof is complete.

\section{Closing Remarks}

We have characterized all 3-by- $n$ TP-completable patterns by finding all minimal obstructions. However, many of the results we used to prove that the list of minimal obstructions is complete do not generalize to 4-by- $n$ patterns. The most notable challenges to solving the 4-by- $n$ case are:

- The word notation does not generalize to 4-by- $n$ patterns since it may be the case that a minimal obstruction has two unspecified entries in the same column. This makes communication about patterns and results much more difficult.

- It is not the case that all 4-by- $n$ patterns with one unspecified entry are TP-completable. In particular, 4-by-4 patterns with one unspecified entry on the antidiagonal are not TP-completable [1].

- Lemma 10's generalization to 4-by- $n$ is much weaker than the 3-by- $n$ case, so proving patterns are TPcompletable is generally more difficult.

- There are substantially more patterns to check for completability. In particular, up to symmetry there are more than 300 4-by-4 patterns that could be minimal obstructions based on the natural extension of the definition of "minimal" to 4-by- $n$ patterns and generalizations to some of the reductions we used in the 3-by- $n$ case.

Acknowledgement: This work was supported by the 2018 National Science Foundation Grant DMS \#0751964.

Data Availability Statement: Data sharing is not applicable to this article as no datasets were generated or analyzed during the current study. 


\section{References}

[1] Shaun Fallat, Charles Johnson, and Ronald Smith. The general totally positive matrix completion problem with few unspecified entries. Electron. J. Linear Algebra, 7:1-20, (2000).

[2] Shaun M. Fallat and Charles R. Johnson. Totally Nonnegative Matrices. Princeton University Press, (2011).

[3] Charles Johnson and David Allen. Doubly constrained totally positive line insertion. Spec. Matrices, 8:181-185, (2020).

[4] Charles Johnson and António Leal-Duarte. On the solvability of derived matrix problems, including completions and duals. Linear Algebra Appl., 514:165-173, (2017).

[5] Duo Wang. TP Matrices and TP Completability. Undergraduate Honors Theses, College of William and Mary, (2018). 